\title{
Comparison of the NIST and PTB Air-Kerma Standards for Low-Energy X-Rays
}

\section{Michelle O'Brien}

National Institute of Standards and Technology, Gaithersburg, MD 20899

and

\section{Ludwig Bueermann}

Physikalisch-Technische

Bundesanstalt, Braunschweig, Germany

michelle.obrien@nist.gov ludwig.bueerman@ptb.de
A comparison has been made of the air-kerma standards for low-energy $\mathrm{x}$ rays at the National Institute of Standards and Technology (NIST) and the PhysikalischTechnische Bundesanstalt (PTB). The comparison involved a series of measurements at the PTB and the NIST using the air-kerma standards and two NIST reference-class transfer ionization chamber standards. Results are presented for the reference radiation beam qualities in the range from $25 \mathrm{kV}$ to $50 \mathrm{kV}$ for low energy $\mathrm{x}$ rays, including the techniques used for mammography dose traceability. The tungsten generated reference radiation qualities, between $25 \mathrm{kV}$ and $50 \mathrm{kV}$ used for this comparison, are new to NIST; therefore this comparison will serve as the preliminary comparison for NIST and a verification of the primary standard correction factors. The mammography comparison will repeat two previously unpublished comparisons between PTB and NIST. The results show the standards to be in reasonable agreement within the standard uncertainty of the comparison of about $0.4 \%$.

Key words: air kerma; free-air ionization chamber; mammography qualities; primary standard; reference radiation qualities; $\mathrm{x}$-ray calibration; $\mathrm{x}$-rays.

Accepted: September 9, 2009

Available online: http://www.nist.gov/jres

\section{Introduction}

An indirect comparison was made between the airkerma primary standards for low-energy $\mathrm{x}$ rays of the National Institute of Standards and Technology (NIST) and the Physikalisch-Technische Bundesanstalt (PTB). The measurements were conducted at both laboratories between January 2006 and September 2008 using the tungsten reference radiation qualities in the range from $10 \mathrm{kV}$ to $50 \mathrm{kV}$ recommended by the Consultative Committee for Ionizing Radiation (CCRI) [1] and various mammography qualities. Two NIST referenceclass ionization chambers were used as transfer instruments for the comparison, one of which has been used for measurement comparisons between NIST and PTB for mammography twice between 1995 and the present.

\section{Determination of the Air-Kerma Rate}

For a free-air ionization chamber standard with measuring volume $V$, the air-kerma rate, $\dot{K}$, is determined by the equation

$$
\dot{K}=\frac{I}{\rho_{\text {air }} V} \frac{W_{\text {air }}}{e} \frac{1}{1-g_{\text {air }}} \prod_{i} k_{i},
$$

where

$I / \rho_{\text {air }} V \quad$ is the mass ionization current measured by the standard,

$W_{\text {air }} \quad$ is the mean energy expended by an electron of charge $e$ to produce an ion pair in dry air, 
$g_{\text {air }} \quad$ is the fraction of the initial electron energy lost by bremsstrahlung production in air, and

$\Pi k_{\mathrm{i}} \quad$ is the product of the correction factors to be applied to the standard.

The values for the physical constants used in the determination of the air-kerma rate are given in Table 1.

Table 1. Physical constants used in the determination of the airkerma rate

\begin{tabular}{ccc}
\hline $\begin{array}{c}\text { Physical } \\
\text { constant }\end{array}$ & Value & $\begin{array}{c}\text { Relative standard } \\
\text { uncertainty } \\
(\%)\end{array}$ \\
\hline$\rho_{\text {air }}{ }^{\text {a }}$ & $1.293 \mathrm{~kg} \mathrm{~m}^{-3}$ & 0.01 \\
$W_{\text {air }} / e$ & $33.97 \mathrm{~J} \mathrm{C}^{-1}$ & 0.15 \\
$1-g_{\text {air }}$ & 1.0000 & 0.01 \\
\hline
\end{tabular}

${ }^{\mathrm{a}}$ Density of dry air at $273.15 \mathrm{~K}$ and $101325 \mathrm{~Pa}$.

\section{Characteristics of Chambers}

\subsection{Description of Air-Kerma Standards}

Two of the air-kerma standard chambers used in the comparison are parallel-plate free-air ionization chambers and the third standard is a variable volume cylindrical chamber. The measuring volume $V$ is defined by the diameter of the chamber aperture and the length of the collecting plate. The PTB air-kerma standard is described in Ref. [2]. The NIST Ritz and Attix chambers are described in Refs. [3-6]. The main dimensions, the measuring volume and the polarizing voltage for each chamber are given in Table $2 \mathrm{a}$.

\subsection{Description of Transfer Ionization Chambers}

Two NIST transfer chambers were used for the comparison. The chambers used for the low-energy $\mathrm{x}$ rays were the Exradin A15 SN 103 and the Exradin A11 SN 114. Each was calibrated at the NIST before and after a series of calibrations at the PTB between January 2006 and September 2008. The transfer standards are constructed with a wall of air-equivalent plastic. Table $2 \mathrm{~b}$ contains the physical characteristics of the transfer chambers. A collecting voltage of $300 \mathrm{~V}$ was applied to each standard. Measurements were made using both polarities at each laboratory.

\section{Comparison Details}

\subsection{Irradiation Facilities and Reference Radiation Qualities}

The radiation qualities in the energy range between $10 \mathrm{kV}$ and $50 \mathrm{kV}$ are those recommended by CCRI [1] and are given in Table $3 \mathrm{a}$ for both NIST and PTB. NIST developed the NIST equivalent CCRI reference radiation qualities to allow for direct comparisons with the Bureau International des Poids et Mesures (BIPM). In past comparisons, the NIST had to interpolate values for radiation qualities with the same energy but different filtration and half-value layers (HVL). The parameters of the mammography reference radiation qualities are listed in Table $3 \mathrm{~b}$. The four reference radiation qualities which are not supported by the standards used in this comparison both at the PTB and the NIST calibration facilities are the NIST qualities at $23 \mathrm{kV}$ and $40 \mathrm{kV}$ and the PTB qualities at $10 \mathrm{kV}$ and $20 \mathrm{kV}$. The associated data for these techniques are included in this report for documentation completeness; however no comparison results are presented. Initially the $10 \mathrm{kV}$ reference

Table 2a. Main characteristics of the primary standards used in the comparison

\begin{tabular}{lllc}
\hline \multicolumn{1}{c}{ Characteristic } & NIST & NIST & PTB \\
& Ritz & Attix 100 \\
\hline Air-path length $/ \mathrm{cm}$ & 12.74 & 21.27 & 9.72 \\
Plate separation $/ \mathrm{cm}$ & 9.0 & Variable & 23.4 \\
Collecting plate length $/ \mathrm{cm}$ & 7.003 & Variable & 2.0021 \\
Aperture diameter $/ \mathrm{cm}$ & 1.0 & 1.0 or 0.5 & 2.0008 \\
Measuring volume $/ \mathrm{cm}^{3}$ & 5.502 & Variable & 6.2947 \\
Polarizing voltage $/ \mathrm{V}$ & -5000 & -2500 & 6000 \\
\hline
\end{tabular}


Volume 114, Number 6, November-December 2009

Journal of Research of the National Institute of Standards and Technology

Table 2b. Main characteristics of ionization chambers used as transfer standards

\begin{tabular}{ccccc}
\hline Model & Serial number & Type & Sensitive volume & Collector diameter \\
\hline A11 & 114 & PLANAR & $0.62 \mathrm{~cm}^{3}$ & $20 \mathrm{~mm}$ \\
A15 & 103 & PLANAR & $2.46 \mathrm{~cm}^{3}$ & $20 \mathrm{~mm}$ \\
\hline
\end{tabular}

Table 3a. Characteristics of the reference radiation qualities used for the comparison at a measurement position of $50 \mathrm{~cm}$

\begin{tabular}{|c|c|c|c|c|c|}
\hline $\begin{array}{l}\text { Reference } \\
\text { radiation }\end{array}$ & $\begin{array}{c}\text { Gernerating } \\
\text { potential } \\
\mathrm{kV}\end{array}$ & $\begin{array}{l}\text { Additional } \\
\text { mm A1 }\end{array}$ & $\begin{array}{l}\text { filtration } \\
\mathrm{mm} \mathrm{Cu}\end{array}$ & $\begin{array}{c}\text { Half-value } \\
\text { layer (HVL) } \\
\text { mm A1 }\end{array}$ & $\begin{array}{c}\text { Air-kerma } \\
\text { rate } \\
\mathrm{mGy} \mathrm{s}^{-1}\end{array}$ \\
\hline \multicolumn{6}{|l|}{ РTB } \\
\hline BIPM10 & 10 & 0 & & 0.033 & 0.28 \\
\hline BIPM25 & 25 & 0.374 & & 0.239 & 0.28 \\
\hline BIPM30 & 30 & 0.208 & & 0.163 & 0.42 \\
\hline BIPM50a & 50 & 4.00 & & 2.291 & 0.19 \\
\hline BIPM50b & 50 & 1.00 & & 1.065 & 0.28 \\
\hline \multicolumn{6}{|l|}{ NIST } \\
\hline BIPM10 & 10 & 0 & & 0.037 & 2.60 \\
\hline BIPM25 & 25 & 0.373 & & 0.240 & 1.40 \\
\hline ВIPM30 & 30 & 0.203 & & 0.167 & 4.20 \\
\hline BIPM40 & 40 & 3.97 & 0.208 & 2.649 & 0.043 \\
\hline BIPM50a & 50 & 3.97 & & 2.291 & 0.730 \\
\hline BIPM50b & 50 & 1.07 & & 1.038 & 3.20 \\
\hline
\end{tabular}

technique was to be included in the comparison results, but in the course of this comparison it was determined that the Ritz chamber is unsuitable for the soft $10 \mathrm{kV}$ reference radiation quality. A different NIST primary standard, the Lamperti chamber [7] has been previously compared [8] at the BIPM providing traceability for $10 \mathrm{kV}$. Due to a new measurement capability at NIST, the Lamperti chamber will be primarily used in a different facility. The Lamperti standard was not included in this comparison.

The NIST x-ray measurements were made using primary standard free-air ionization chambers, the Ritz in the NIST $100 \mathrm{kV}$ tungsten calibration facility range and the Attic in the mammography facility. For the NIST $100 \mathrm{kV}$ range, the $\mathrm{x}$-ray source is a $100 \mathrm{kV} \mathrm{x}$-ray generator with a metal-ceramic $x$-ray tube, supplied by
Pantak and Comet ${ }^{1}$. In the NIST mammography facility the X-ray source is a Gulmay generator and a Lohmen Mo anode tube. Both X-ray generators are high-frequency, highly stabilized voltage sources. Both tubes have a $1 \mathrm{~mm}$ beryllium window. The materials used for the filtration and for the measurement of HVL were at least $99.99 \%$ pure with thicknesses known with an uncertainty of $0.01 \mathrm{~mm}$. The high voltage was verified through the use of an invasive voltage divider custom made by Pantak.

\footnotetext{
${ }^{1}$ Certain commercial equipment, instruments, or materials are identified in this paper to foster understanding. Such identification does not imply recommendation or endorsement by the National Institute of Standards and Technology, nor does it imply that the materials or equipment identified are necessarily the best available for the purpose
} 
Table 3b. Characteristics of the mammography reference radiation qualities used for the comparison at a measurement position of $100 \mathrm{~cm}$

\begin{tabular}{|c|c|c|c|c|}
\hline $\begin{array}{c}\text { Reference } \\
\text { radiation }\end{array}$ & $\begin{array}{c}\text { Tube } \\
\text { voltage } \\
(\mathrm{kV})\end{array}$ & $\begin{array}{l}\text { Additional filtration } \\
\qquad(\mathrm{mm})\end{array}$ & $\begin{array}{l}\text { Half-value } \\
\text { layer (HVL) } \\
\text { (mm A1) }\end{array}$ & $\begin{array}{l}\text { Air-kerma } \\
\text { rate } \\
\mathrm{mGy} \mathrm{s}^{-1}\end{array}$ \\
\hline \multicolumn{5}{|l|}{ РTB } \\
\hline MMV 20 & 20 & 0.03 Mo & 0.22 & 0.555 \\
\hline MMV 25 & 25 & 0.03 Mo & 0.29 & 0.108 \\
\hline MMV 28 & 28 & 0.03 Mo & 0.32 & 1.070 \\
\hline MMV 30 & 30 & $0.03 \mathrm{Mo}$ & 0.33 & 1.030 \\
\hline MMV 35 & 35 & $0.03 \mathrm{Mo}$ & 0.37 & 1.070 \\
\hline MMH 20 & 20 & $0.030 \mathrm{Mo}+2.0 \mathrm{Al}$ & 0.46 & 0.013 \\
\hline MMH 25 & 25 & $0.030 \mathrm{Mo}+2.0 \mathrm{Al}$ & 0.56 & 0.062 \\
\hline MMH 28 & 28 & $0.030 \mathrm{Mo}+2.0 \mathrm{Al}$ & 0.61 & 0.069 \\
\hline MMH 30 & 30 & $0.030 \mathrm{Mo}+2.0 \mathrm{Al}$ & 0.64 & 0.071 \\
\hline MMH 35 & 35 & $0.030 \mathrm{Mo}+2.0 \mathrm{Al}$ & 0.73 & 0.070 \\
\hline \multicolumn{5}{|l|}{ NIST } \\
\hline $\mathrm{Mo} / \mathrm{Mo} 23$ & 23 & $0.032 \mathrm{Mo}$ & 0.271 & 0.330 \\
\hline Mo/Mo25 & 25 & $0.032 \mathrm{Mo}$ & 0.296 & 0.460 \\
\hline $\mathrm{Mo} / \mathrm{Mo} 28$ & 28 & $0.032 \mathrm{Mo}$ & 0.332 & 0.680 \\
\hline Mo/Mo30 & 30 & $0.032 \mathrm{Mo}$ & 0.351 & 0.400 \\
\hline Mo/Mo35 & 35 & $0.032 \mathrm{Mo}$ & 0.392 & 0.840 \\
\hline $\mathrm{Mo} / \mathrm{Mo} 25 \mathrm{x}$ & 25 & $0.030 \mathrm{Mo}+2.0 \mathrm{Al}$ & 0.566 & 0.018 \\
\hline $\mathrm{Mo} / \mathrm{Mo} 28 \mathrm{x}$ & 28 & $0.030 \mathrm{Mo}+2.0 \mathrm{Al}$ & 0.626 & 0.038 \\
\hline Mo/Mo30x & 30 & $0.030 \mathrm{Mo}+2.0 \mathrm{Al}$ & 0.660 & 0.055 \\
\hline $\mathrm{Mo} / \mathrm{Mo} 35 \mathrm{x}$ & 35 & $0.030 \mathrm{Mo}+2.0 \mathrm{Al}$ & 0.748 & 0.120 \\
\hline
\end{tabular}

The PTB x-ray air kerma measurements were made using the PK100 primary standard free-air ionization chamber. The PTB mammographic reference radiation qualities are produced with a unipolar $\mathrm{x}$-ray tube of type Philips PW2185/Mo with a Mo anode angle of $26^{\circ}$ combined with a constant potential generator. The in-herent filtration is $1 \mathrm{~mm}$ beryllium and the molybdenum filtration is $0.03 \mathrm{~mm}$. Radiation qualities are established for tube voltages in the range from $20 \mathrm{kV}$ to $50 \mathrm{kV}$. The PTB CCRI radiation qualities are also realized with a unipolar x-ray tube of type Comet MXR 160/0.4-3.0 with a $\mathrm{W}$ anode angle of $20^{\circ}$ combined with a constant potential generator. The inherent filtration is $1 \mathrm{~mm}$ beryllium. For both x-ray facilities the high voltage was measured invasively with a voltage divider manufactured and calibrated at PTB. A transmission-type monitor chamber manufactured at TB was used at both facilities to normalize the x-ray output.

\subsection{Correction Factors}

Although free-air chambers are designed to minimize or eliminate corrections to the measured ionization current, certain corrections are unavoidable. The correction factors applied to each free-air chamber and the associated uncertainties are listed in Tables 4 to $6 \mathrm{c}$. A correction must be made for the attenuation of the $\mathrm{x}$-ray fluence along the air path between the reference plane and the center of the collecting volume. The correction factor $k_{\mathrm{a}}$ is calculated using the measured air-attenuation coefficients $\mu_{\text {air }}$, according to

$$
k_{\mathrm{a}}=\exp \left(\mu_{\text {air }} L\right) .
$$

The effective attenuation path $L$ varies with the temperature and pressure of the air in the chamber and so the values for $k_{\mathrm{a}}$ are corrected for this effect. All ionization measurements are also corrected for the temperature 
and pressure of the ambient air between the radiation source and the reference plane.

All measured ionization currents using the free-air chamber standards are corrected for ion recombination, $k_{\mathrm{s}}$. The ionization currents measured with the transfer standards are not corrected for ion recombination.
However, since the air-kerma rates used at both facilities are low, minimal volume recombination occurs in the transfer chambers. The standard chambers are corrected for the humidity effect $k_{\mathrm{h}}$, which is taken as 0.998 for NIST and PTB standards for all reference radiation qualities.

Table 4. Correction factors used in the comparison for the NIST Ritz standard

\begin{tabular}{|c|c|c|c|c|c|c|c|}
\hline \multirow[t]{2}{*}{ Correction factor } & \multicolumn{5}{|c|}{ Generating potential $(\mathrm{kV})$} & \multicolumn{2}{|c|}{$\begin{array}{l}\text { Relative standard } \\
\text { uncertainty }\end{array}$} \\
\hline & 25 & 30 & 40 & $50 \mathrm{a}$ & $50 \mathrm{~b}$ & Type A & Type B \\
\hline Air attenuation $k_{\mathrm{a}}^{\mathrm{a}}$ & 1.039 & 1.054 & 1.005 & 1.006 & 1.012 & 0.01 & 0.01 \\
\hline Scattered radiation $k_{\mathrm{sc}}$ & 0.9966 & 0.9967 & 0.9976 & 0.9974 & 0.9952 & & 0.07 \\
\hline Electron loss $k_{\mathrm{e}}$ & 1.000 & 1.000 & 1.000 & 1.005 & 1.001 & & 0.05 \\
\hline Ion recombination $k_{\mathrm{s}}$ & 1.000 & 1.000 & 1.000 & 1.000 & 1.000 & 0.03 & \\
\hline Fluorescence $k_{\mathrm{f} 1}$ & 0.9961 & 0.9962 & 0.99828 & 0.9981 & 0.9976 & & 0.03 \\
\hline Aperture transmission $k_{1}$ & 1.000 & 1.000 & 1.000 & 1.000 & 1.000 & & 0.04 \\
\hline Field distortion $k_{\mathrm{d}}$ & 1.000 & 1.000 & 1.000 & 1.000 & 1.000 & & 0.1 \\
\hline Wall transmission $k_{\mathrm{p}}$ & 1.000 & 1.000 & 1.000 & 1.000 & 1.000 & & 0.01 \\
\hline Humidity $k_{\mathrm{h}}$ & 0.998 & 0.998 & 0.998 & 0.998 & 0.998 & & 0.03 \\
\hline
\end{tabular}

Table 5a. Correction factors used in the comparison for the NIST Attix mammography standard

\begin{tabular}{|c|c|c|c|c|c|c|c|}
\hline \multirow[t]{2}{*}{ Correction factor } & \multicolumn{5}{|c|}{ Reference radiation qualities } & \multicolumn{2}{|c|}{$\begin{array}{l}\text { Relative standard } \\
\text { uncertainty }(\%)\end{array}$} \\
\hline & Mo / Mo23 & Mo / Mo 25 & $\mathrm{Mo} / \mathrm{Mo} 28$ & Mo/Mo30 & Mo / Mo35 & Type A & Type B \\
\hline Air attenuation $k_{\mathrm{a}}^{\mathrm{a}}$ & 1.055 & 1.051 & 1.047 & 1.043 & 1.040 & & 0.01 \\
\hline Scattered radiation $k_{\mathrm{sc}}$ & 0.9949 & 0.9950 & 0.9950 & 0.9951 & 0.9952 & & 0.07 \\
\hline Electron loss $k_{\mathrm{e}}$ & 1.000 & 1.000 & 1.000 & 1.000 & 1.000 & & 0.05 \\
\hline Ion recombination $k_{\mathrm{s}}$ & 1.000 & 1.000 & 1.000 & 1.000 & 1.000 & 0.04 & \\
\hline Humidity $k_{\mathrm{h}}$ & 0.998 & 0.998 & 0.998 & 0.998 & 0.998 & & 0.03 \\
\hline
\end{tabular}

Table 5b. Correction factors used in the comparison for the NIST Attix mammography standard

\begin{tabular}{|c|c|c|c|c|c|c|}
\hline \multirow[t]{2}{*}{ Correction factor } & \multicolumn{4}{|c|}{ Reference radiation qualities } & \multicolumn{2}{|c|}{$\begin{array}{l}\text { Relative standard } \\
\text { uncertainty }(\%)\end{array}$} \\
\hline & Mo/Mo25x & Mo / Mo28x & Mo/Mo30x & Mo/Mo35x & Type A & Type B \\
\hline Air attenuation $k_{\mathrm{a}}^{\mathrm{a}}$ & 1.021 & 1.024 & 1.021 & 1.020 & & 0.01 \\
\hline Scattered radiation $k_{\mathrm{sc}}$ & 0.9955 & 0.9956 & 0.9956 & 0.9957 & & 0.07 \\
\hline Electron loss $k_{\mathrm{e}}$ & 1.000 & 1.000 & 1.000 & 1.000 & & 0.05 \\
\hline Ion recombination $k_{\mathrm{s}}$ & 1.000 & 1.000 & 1.000 & 1.000 & 0.04 & \\
\hline Humidity $k_{\mathrm{h}}$ & 0.998 & 0.998 & 0.998 & 0.998 & & 0.03 \\
\hline
\end{tabular}

a These are nominal values for $T=293.15 \mathrm{~K}$ and $p=101325 \mathrm{~Pa}$. Each measurement is corrected using the air temperature and pressure measured at the time. 


\section{Journal of Research of the National Institute of Standards and Technology}

Table 6a. Correction factors used in the comparison for the PTB PK100 standard: BIPM reference radiation qualities (PTB F-series)

\begin{tabular}{|c|c|c|c|c|c|c|c|}
\hline \multirow[t]{2}{*}{ Correction factor } & \multicolumn{4}{|c|}{ Generating potential $(\mathrm{kV})$} & \multicolumn{3}{|c|}{$\begin{array}{l}\text { Relative standard } \\
\text { uncertainty }(\%)\end{array}$} \\
\hline & 10 & 25 & 30 & $50 \mathrm{a}$ & $50 \mathrm{~b}$ & Type A & Type B \\
\hline Air attenuation $k_{\mathrm{a}}^{\mathrm{a}}$ & 1.2274 & 1.0340 & 1.0485 & 1.0046 & 1.0095 & 0.05 & 0.05 \\
\hline Scattered radiation $k_{\mathrm{sc}}^{\mathrm{b}}$ & 0.9854 & 0.9901 & 0.9893 & 0.9939 & 0.9927 & & 0.05 \\
\hline Electron loss $k_{\mathrm{e}}$ & 1.0000 & 1.0000 & 1.0000 & 1.0000 & 1.0000 & & 0.05 \\
\hline Volume recombination $k_{\mathrm{sv}}$ & 1.0014 & 1.0017 & 1.0026 & 1.0012 & 1.0018 & 0.05 & 0.05 \\
\hline Initial recombination $k_{\mathrm{si}}$ & 1.0005 & 1.0005 & 1.0005 & 1.0005 & 1.0005 & & 0.05 \\
\hline Guard srip attenuation $k_{\text {ap }}$ & 1.0393 & 1.0075 & 1.0110 & 1.0015 & 1.0027 & 0.05 & 0.05 \\
\hline Aperture edge transmission $k_{1}$ & 0.9998 & 0.9991 & 0.9992 & 0.9971 & 0.9979 & & 0.05 \\
\hline Field distortion $k_{\mathrm{d}}$ & 0.9920 & 0.9920 & 0.9920 & 0.9920 & 0.9920 & & 0.15 \\
\hline Wall transmission $k_{\mathrm{p}}$ & 1.0000 & 1.0000 & 1.0000 & 1.0000 & 1.0000 & 0.05 & \\
\hline Humidity $k_{\mathrm{h}}$ & 0.9980 & 0.9980 & 0.9980 & 0.9980 & 0.9980 & & 0.05 \\
\hline
\end{tabular}

Table 6b. Correction factors used in the comparison for the PTB PK100 standard: Mammography radiation qualities, non-attenuated (PTB MMV-series)

\begin{tabular}{|c|c|c|c|c|c|c|c|}
\hline \multirow[t]{2}{*}{ Correction factor } & \multicolumn{5}{|c|}{ Generating potential $(\mathrm{kV})$} & \multicolumn{2}{|c|}{$\begin{array}{c}\text { Relative standard } \\
\text { uncertainty (\%) }\end{array}$} \\
\hline & 20 & 25 & 28 & 30 & 35 & Type A & Type B \\
\hline Air attenuation $k_{\mathrm{a}}^{\mathrm{a}}$ & 1.0333 & 1.0275 & 1.0256 & 1.0250 & 1.0232 & 0.05 & 0.05 \\
\hline Scattered radiation $k_{\mathrm{sc}}{ }^{\mathrm{b}}$ & 0.9899 & 0.9904 & 0.9906 & 0.9907 & 0.9909 & & 0.05 \\
\hline Electron loss $k_{\mathrm{e}}$ & 1.0000 & 1.0000 & 1.0000 & 1.0000 & 1.0000 & & 0.05 \\
\hline Volume recombination $k_{\mathrm{sv}}$ & 1.0001 & 1.0004 & 1.0004 & 1.0005 & 1.0005 & 0.05 & 0.05 \\
\hline Initial recombination $k_{\mathrm{si}}$ & 1.0005 & 1.0005 & 1.0005 & 1.0005 & 1.0005 & & 0.05 \\
\hline Guard srip attenuation $k_{\text {ap }}$ & 1.0068 & 1.0056 & 1.0052 & 1.0051 & 1.0047 & 0.05 & 0.05 \\
\hline Aperture edge transmission $k_{1}$ & 0.9997 & 0.9997 & 0.9996 & 0.9996 & 0.9996 & & 0.05 \\
\hline Field distortion $k_{\mathrm{d}}$ & 0.9920 & 0.9920 & 0.9920 & 0.9920 & 0.9920 & & 0.15 \\
\hline Wall transmission $k_{\mathrm{p}}$ & 1.0000 & 1.0000 & 1.0000 & 1.0000 & 1.0000 & 0.05 & \\
\hline Humidity $k_{\mathrm{h}}$ & 0.9980 & 0.9980 & 0.9980 & 0.9980 & 0.9980 & & 0.05 \\
\hline
\end{tabular}

\footnotetext{
${ }^{\mathrm{a}}$ These are nominal values for $T=293.15 \mathrm{~K}$ and $p=101325 \mathrm{~Pa}$. Each measurement is corrected using the air temperature and pressure measured at the time.

${ }^{\mathrm{b}}$ This correction includes the re-absorption of scattered radiation and of fluorescent photons.
} 
Table 6c. Correction factors used in the comparison for the PTB PK100 standard: Mammography radiation qualities, attenuated (PTB MMH-series)

\begin{tabular}{|c|c|c|c|c|c|c|c|}
\hline \multirow[t]{2}{*}{ Correction factor } & \multicolumn{5}{|c|}{ Generating potential $(\mathrm{kV})$} & \multicolumn{2}{|c|}{$\begin{array}{c}\text { Relative standard } \\
\text { uncertainty }(\%)\end{array}$} \\
\hline & 20 & 25 & 28 & 30 & 35 & Type A & Type B \\
\hline Air attenuation $k_{\mathrm{a}}{ }^{\mathrm{a}}$ & 1.0138 & 1.0116 & 1.0110 & 1.0106 & 1.0098 & 0.05 & 0.05 \\
\hline Scattered radiation $k_{\mathrm{sc}}{ }^{\mathrm{b}}$ & 0.9916 & 0.9919 & 0.9920 & 0.9921 & 0.9923 & & 0.05 \\
\hline Electron loss $k_{\mathrm{e}}$ & 1.0000 & 1.0000 & 1.0000 & 1.0000 & 1.0000 & & 0.05 \\
\hline Volume recombination $k_{\mathrm{sv}}$ & 1.0001 & 1.0004 & 1.0004 & 1.0005 & 1.0005 & 0.05 & 0.05 \\
\hline Initial recombination $k_{\mathrm{si}}$ & 1.0005 & 1.0005 & 1.0005 & 1.0005 & 1.0005 & & 0.05 \\
\hline Guard srip attenuation $k_{\text {ap }}$ & 1.0029 & 1.0024 & 1.0023 & 1.0023 & 1.0021 & 0.05 & 0.05 \\
\hline Aperture edge transmission $k_{1}$ & 0.9996 & 0.9995 & 0.9995 & 0.9994 & 0.9994 & & 0.05 \\
\hline Field distortion $k_{\mathrm{d}}$ & 0.9920 & 0.9920 & 0.9920 & 0.9920 & 0.9920 & & 0.15 \\
\hline Wall transmission $k_{\mathrm{p}}$ & 1.0000 & 1.0000 & 1.0000 & 1.0000 & 1.0000 & 0.05 & \\
\hline Humidity $k_{\mathrm{h}}$ & 0.9980 & 0.9980 & 0.9980 & 0.9980 & 0.9980 & & 0.05 \\
\hline
\end{tabular}

\subsection{Chamber Positioning and Measurement Procedure}

Each calibration was made by alternating between the transfer chambers and the standard free-air chamber for the x-ray measurements. At both laboratories, alignment on the beam axis was measured to an accuracy of around $0.1 \mathrm{~mm}$, and this position was reproducible to better than $0.01 \mathrm{~mm}$ as observed by an alignment telescope. No correction was applied for the radial nonuniformity of any beam. The reference plane for each $\mathrm{X}$-ray standard chamber was positioned at $500 \mathrm{~mm}$ for the tungsten work and $1000 \mathrm{~mm}$ for the mammography work. At NIST, the beam diameter in the reference plane was $70 \mathrm{~mm}$ for the beams measured with the Ritz chamber and $80 \mathrm{~mm}$ for those measured with the Attix chamber. The beam diameter in the reference plane for the PTB x-ray measurements was $83 \mathrm{~mm}$. The background current was measured before and after each series of ionization current measurements and a correction made based on the mean of these leakage measurements. The background current-to-signal ratios varied for the low rate attenuated mammography beams and the higher rate tungsten and unfiltered mammography beams. The background current-to-signal ratios were between $1 \%$ and less than $0.01 \%$. For all $\mathrm{x}$-ray chambers at the NIST, a total of not less than
10 measurement sets with an integration time of $120 \mathrm{~s}$, $90 \mathrm{~s}$ or $60 \mathrm{~s}$ were made, except for the data for the mammography positive charge sets. Due to equipment failure, only two sets of three $120 \mathrm{~s}$ measurements were made for the mammography positive charge data. The standard deviations (Type A uncertainty components) of the calibration coefficient measurements for the CCRI (NIST-BIPM) beams were typically $0.1 \%$ for the NIST transfer chambers. The standard deviations of the calibration coefficient measurements for the NIST mammography beams were typically less than $0.5 \%$ for the NIST transfer chambers. The measurement procedure at PTB is similar to those at NIST. The leakage charge was measured before and after a series of five repeated charge measurements with the x-ray beam turned on and the mean of both leakage charge measurements was subtracted. The integration times were $60 \mathrm{~s}$ and $100 \mathrm{~s}$ depending on the actual chamber currents. A transmission-type monitor chamber manufactured at PTB was used to normalize the x-ray output. The standard deviations of the calibration coefficients of both transfer chambers were always less than $0.1 \%$. The leakage-to-signal ratio of the A11 and A15 chamber was less than $0.5 \%$ and $0.2 \%$ in the attenuated mammography beams, respectively, and less than $0.1 \%$ in every other case. 
All transfer ionization chamber current measurements were normalized to $293.15 \mathrm{~K}$ and $101325 \mathrm{~Pa}$. The transfer chamber currents were not corrected for humidity. The humidity is monitored and recorded at both facilities. The PTB laboratory humidity is not controlled because it varies by no more than between $30 \%$ and $60 \%$ and this is taken into account by an additional uncertainty in the humidity correction factor. The NIST laboratory average humidity is $30 \%$.

\section{Measurement Uncertainties}

The uncertainties associated with the primary standards are given in Table 7 . The NIST uncertainties were evaluated according to Ref. [9]. The uncertainties associated with the calibration of the transfer ionization chambers at the NIST and at the PTB are listed in Table 8. The uncertainty of the comparison results is given in Table 9. The comparison uncertainty calculation removed the correlations due to Type B uncertainties from the physical constants and the correction for humidity. Correlations also exist in the values for $k_{\mathrm{e}}, k_{\mathrm{sc}}$ and $k_{\mathrm{fl}}$, since both laboratories use values derived from the same Monte Carlo calculations [10]. These are accounted for by taking half the uncertainty value for each component at each laboratory, in accordance with the analysis made for the degrees of equivalence appearing in Ref. 11. All other correction factors are assumed to be uncorrelated.

Table 7. Relative standard uncertainties (in \%) associated with the standards

\begin{tabular}{|c|c|c|c|c|c|c|}
\hline \multirow[t]{2}{*}{ Source of uncertainty } & \multicolumn{2}{|c|}{ NIST Attix } & \multicolumn{2}{|c|}{ NIST Ritz } & \multicolumn{2}{|c|}{ PTB } \\
\hline & Type A & Type B & Type A & Type B & Type A & Type B \\
\hline Ionization current & 0.13 & 0.06 & 0.05 & 0.06 & 0.10 & 0.06 \\
\hline Volume & 0.01 & 0.07 & 0.04 & 0.01 & & 0.06 \\
\hline Positioning & & 0.01 & & 0.01 & & 0.01 \\
\hline Correction factors (excl. $k_{\mathrm{h}}$ ) & 0.04 & 0.09 & 0.03 & 0.14 & 0.10 & 0.20 \\
\hline Physical constants & & 0.15 & & 0.15 & & 0.15 \\
\hline \multirow{2}{*}{$\dot{K}_{\mathrm{LAB}}$} & 0.14 & 0.20 & 0.07 & 0.22 & 0.14 & 0.26 \\
\hline & \multicolumn{2}{|c|}{0.24} & \multicolumn{2}{|c|}{0.23} & \multicolumn{2}{|c|}{0.30} \\
\hline
\end{tabular}

Table 8. Relative standard uncertainties (in \%) associated with the calibration of the transfer ionization chambers

\begin{tabular}{|c|c|c|c|c|c|c|}
\hline \multirow[t]{2}{*}{ Source of uncertainty } & \multicolumn{2}{|c|}{ NIST Attix } & \multicolumn{2}{|c|}{ NIST Ritz } & \multicolumn{2}{|c|}{ РTB } \\
\hline & Type A & Type B & Type A & Type B & Type A & Type B \\
\hline Air-kerma rate $\dot{K}_{\mathrm{LAB}}$ & 0.14 & 0.20 & 0.07 & 0.22 & 0.14 & 0.26 \\
\hline Ionization current & 0.09 & 0.06 & 0.05 & 0.06 & 0.10 & 0.06 \\
\hline Positioning & & 0.01 & 0.01 & & & 0.04 \\
\hline Monitor normalization & & & & & & 0.05 \\
\hline Air density correction & & & & & & 0.04 \\
\hline \multirow{2}{*}{$N_{K, \mathrm{LAB}}$} & 0.17 & 0.21 & 0.09 & 0.23 & 0.17 & 0.28 \\
\hline & \multicolumn{2}{|c|}{0.27} & \multicolumn{2}{|c|}{0.24} & \multicolumn{2}{|c|}{0.33} \\
\hline
\end{tabular}


Table 9. Relative standard uncertainties (in \%) associated with the comparison results $R_{K}$

\begin{tabular}{lcc}
\hline \hline Source of uncertainty & Type A & Type B \\
\hline & 0.24 & 0.28 \\
Comparison result $R_{K}$ & & \\
& & 0.37 \\
\hline
\end{tabular}

\section{Results and Discussion}

The results for the transfer ionization chamber calibrations at both laboratories are shown in Tables $10 \mathrm{a}$ and $10 \mathrm{~b}$. The average of the calibration coefficients collected at both polarities was determined for each chamber and then used to determine the comparison ratios. For each transfer chamber and each radiation quality, the ratio of the calibration coefficients $N_{K \text {, NIST }} / N_{K \text {, PTB }}$ is evaluated, as shown in Table 11 , as well as the mean of the ratios. Comparison results are not included for the $10 \mathrm{kV}$ technique, because it was verified through the course of the comparison that the Ritz chamber, as currently used at NIST with the $7 \mathrm{~cm}$ collecting plate, is not well suited for the soft $10 \mathrm{kV}$ reference radiation qualities. Although correction factors have previously been determined for the $10 \mathrm{kV}$ beam using the Ritz chamber, more work would be required to determine the appropriateness of the replacement of the Lamperti chamber with the Ritz chamber for measurement traceability at $10 \mathrm{kV}$. The agreement of the Ritz to the PTB PK100 standard for $10 \mathrm{kV}$ is on the order of $2.5 \%$, which is the same agreement previously determined between the two NIST low energy standards, the Lamperti and the Ritz chamber. This disagreement has never been a concern because until recently the Lamperti chamber was readily available to provide traceability for $10 \mathrm{kV}$. The $10 \mathrm{kV}$ reference techniques at NIST are generally only used for comparison measurement work at NIST and rarely requested for routine calibrations. Unless there is increased demand or interest in the $10 \mathrm{kV}$ technique, no further investigation into the limitations of the Ritz chamber will transpire.

Table 10a. Measured PTB results for the calibration of the NIST transfer chambers at both laboratories

\begin{tabular}{|c|c|c|c|c|}
\hline \multirow{2}{*}{$\begin{array}{l}\text { Reference radiation } \\
\text { (polarity of charge) }\end{array}$} & \multicolumn{4}{|c|}{$\begin{array}{l}\text { NIST transfer chambers } \\
\text { calibration coefficients } \\
\qquad\left(10^{7} \mathrm{~Gy} \mathrm{C}^{-1}\right)\end{array}$} \\
\hline & $\begin{array}{c}\text { A11 SN114 } \\
\text { (negative) }\end{array}$ & $\begin{array}{c}\text { A11 SN114 } \\
\text { (positive) }\end{array}$ & $\begin{array}{c}\text { A11 SN114 } \\
\text { (negative) }\end{array}$ & $\begin{array}{c}\text { A11 SN114 } \\
\text { (positive) }\end{array}$ \\
\hline BIPM10 & 3.326 & 3.315 & 1.341 & 1.347 \\
\hline BIPM25 & 3.268 & 3.245 & 1.259 & 1.265 \\
\hline BIPM30 & 3.262 & 3.243 & 1.269 & 1.277 \\
\hline BIPM50a & 3.203 & 3.162 & 1.207 & 1.213 \\
\hline BIPM50b & 3.247 & 3.210 & 1.221 & 1.226 \\
\hline MMV 20 & 3.259 & 3.239 & 1.254 & 1.263 \\
\hline MMV 25 & 3.267 & 3.245 & 1.245 & 1.254 \\
\hline MMV 28 & 3.271 & 3.250 & 1.243 & 1.251 \\
\hline MMV 30 & 3.273 & 3.252 & 1.242 & 1.251 \\
\hline MMV 35 & 3.276 & 3.254 & 1.239 & 1.248 \\
\hline MMH 20 & 3.293 & 3.250 & 1.235 & 1.240 \\
\hline MMH 25 & 3.281 & 3.250 & 1.225 & 1.233 \\
\hline MMH 28 & 3.278 & 3.249 & 1.222 & 1.230 \\
\hline MMH 30 & 3.274 & 3.245 & 1.221 & 1.228 \\
\hline MMH 35 & 3.268 & 3.240 & 1.218 & 1.226 \\
\hline
\end{tabular}


Volume 114, Number 6, November-December 2009

Journal of Research of the National Institute of Standards and Technology

Table 10b. Measured PTB results for the calibration of the NIST transfer chambers at both laboratories

\begin{tabular}{|c|c|c|c|c|}
\hline \multirow{2}{*}{$\begin{array}{l}\text { Reference radiation } \\
\text { (polarity of charge) }\end{array}$} & \multicolumn{4}{|c|}{$\begin{array}{l}\text { NIST transfer chambers } \\
\text { calibration coefficients } \\
\qquad\left(10^{7} \mathrm{~Gy} \mathrm{C}^{-1}\right)\end{array}$} \\
\hline & $\begin{array}{c}\text { A11 SN114 } \\
\text { (negative) }\end{array}$ & $\begin{array}{c}\text { A11 SN114 } \\
\text { (positive) }\end{array}$ & $\begin{array}{c}\text { A11 SN114 } \\
\text { (negative) }\end{array}$ & $\begin{array}{c}\text { A11 SN114 } \\
\text { (positive) }\end{array}$ \\
\hline BIPM25 & 3.260 & 3.245 & 1.258 & 1.262 \\
\hline BIPM30 & 3.247 & 3.231 & 1.260 & 1.269 \\
\hline BIPM40 & 3.202 & 3.186 & 1.209 & 1.218 \\
\hline BIPM50a & 3.207 & 3.183 & 1.209 & 1.216 \\
\hline BIPM50b & 3.262 & 3.237 & 1.225 & 1.232 \\
\hline Mo/Mo23 & 3.264 & 3.252 & 1.247 & 1.262 \\
\hline Mo/Mo25 & 3.267 & 3.246 & 1.244 & 1.254 \\
\hline Mo/Mo28 & 3.260 & 3.248 & 1.250 & 1.252 \\
\hline Mo/Mo30 & 3.271 & 3.246 & 1.240 & 1.245 \\
\hline Mo/Mo35 & 3.270 & 3.242 & 1.243 & 1.242 \\
\hline Mo/Mo25x & 3.291 & 3.269 & 1.234 & 1.240 \\
\hline Mo/Mo28x & 3.293 & 3.268 & 1.227 & 1.236 \\
\hline Mo/Mo30x & 3.291 & 3.261 & 1.227 & 1.229 \\
\hline Mo/Mo35x & 3.262 & 3.249 & 1.219 & 1.230 \\
\hline
\end{tabular}

Table 11. Results of the comparison for each of the transfer chambers shown as the ratio of the average calibration coefficient $N_{\mathrm{K}, \mathrm{NIST}} / N_{\mathrm{K} \text {, PTB }}$ for both chambers, as well as the mean

\begin{tabular}{lllc}
\hline \hline Reference radiation & A11 & A15 & Mean \\
& SN114 & SN103 & $N_{K, \text { NIST }} /$ \\
& & & $N_{K, \text { PTB }}$ \\
\hline BIPM25 & 0.999 & 0.998 & 0.998 \\
BIPM30 & 0.996 & 0.993 & 0.995 \\
BIPM50a & 1.004 & 1.002 & 1.003 \\
BIPM50b & 1.007 & 1.004 & 1.005 \\
Mo/Mo25 & 1.000 & 0.999 & 1.000 \\
Mo/Mo28 & 0.998 & 1.003 & 1.001 \\
Mo/Mo30 & 0.999 & 0.997 & 0.998 \\
Mo/Mo35 & 0.997 & 0.999 & 0.998 \\
Mo/Mo25x & 1.004 & 1.006 & 1.005 \\
Mo/Mo28x & 1.005 & 1.005 & 1.005 \\
Mo/Mo30x & 1.005 & 1.003 & 1.004 \\
Mo/Mo35x & 1.000 & 1.002 & 1.001 \\
\hline
\end{tabular}


Agreement of $0.5 \%$ can be achieved for the reference radiation qualities used for this comparison. Agreement of $0.2 \%$ was achieved for the $0.03 \mathrm{~mm}$ Mo filtered mammography techniques, while the mammography reference radiation qualities filtered with the $2 \mathrm{~mm}$ of aluminum compared on the $0.5 \%$ level. The comparison results for mammography techniques demonstrated consistency between NIST and PTB from the previous two series of unpublished measurements performed since 1995. The comparison results for the $25 \mathrm{kV}, 30 \mathrm{kV}$ and $50 \mathrm{kV}$ tungsten reference radiation qualities serve as verification to the changes and additions to the reference radiation qualities maintained at the NIST. The correction factors for the Ritz chamber will be verified and perhaps reevaluated through some calculations supported by recently acquired spectral measurements.

\section{References}

[1] BIPM, Qualités de rayonnements, Consultative Committee for Ionizing Radiation (CCEMRI) (I), 1972, R15.

[2] Engelke B. A., Oetzmann W., and Struppek, G., Die Meßeinrichtungen der Physikalisch-Technischen Bundesanstalt zur Darstellung der Einheiten der Standard-Ionendosis, PhotonenÄquivalentdosis und Luftkerma, PTB-Report Dos-16, 1988 (Physikalisch-Technische Bundesanstalt, Braunschweig).

[3] H. O. Wyckoff and F. H. Attix, Design of free-air ionization chambers, National Bureau of Standards Handbook 64, (1957).

[4] P. J. Lamperti and M. O'Brien, Calibration of X-Ray and Gamma-Ray Measuring Instruments, NIST Special Publication 250-58, (2001).

[5] F. H. Attix, Electronic equilibrium in free-air chambers and a proposed new chamber design, Naval Research Laboratory Report No. 5646, 1961.

[6] J. G. Coletti, D. W. Pearson, and L.A. DeWerd, Mammography exposure standards: Design and characterization of free-air ionization chamber, Rev. Sci Instrum. 66, 2574-2577 (1995).

[7] P. J. Lamperti and H. O.Wyckoff, NBS free-air chamber for measurement of 10 to $60 \mathrm{kV}$ x rays, J. Res. Nat. Bur. Stand. (U.S.), 69C, 39-47 (1965).

[8] D. T. Burns, P. Lamperti, and M. O'Brien, Comparison of the NIST and BIPM Air-Kerma Standards for Measurements in the Low-Energy X-Ray Range, J. Res. Natl. Inst. Stand.Technol. 104, 135 (1999).

[9] B. N. Taylor and C. E. Kuyatt, Guidelines for Evaluating and Expressing the Uncertainty of NIST Measurement Results, 1994 Edition, NIST Technical Note 1297, September 1994.

[10] D. T. Burns, Free-air chamber correction factors for electron loss, photon scatter, fluorescence and bremsstrahlung, CCRI(I)/01-36, 2001.

[11] D. T. Burns, Degrees of equivalence for the key comparison BIPM.RI(I)-K3 between national standards for mediumenergy x rays, Metrologia 40, Technical Supplement, 06036 (2003).
About the authors: Michelle O'Brien is a physicist in the Ionizing Radiation Division, Radiation Interactions and Dosimetry Group of the NIST Physics Laboratory. Ludwig Bueermann is a physicist in the Ionizing Radiation Division of the PTB. Both authors maintain the primary $x$-ray standards at their institutions while conducting comparisons between other national measurement institutions and facilitating $x$-ray dose measurement quality standards domestically. Both are experts in $x$-ray dose traceability. The National Institute of Standards and Technology is an agency of the U.S. Department of Commerce. 